Supporting Information for:

\title{
Synthesis, Crystal structure and Transistor Performance of Tetracene Derivatives
}

\author{
Hyunsik Moon,,$^{\dagger}$ Roswitha Zeis, ${ }^{\dagger}$ Evert-Jan Borkent, ${ }^{\dagger}$ Celine Besnard, ${ }^{\ddagger}$ Andrew J. Lovinger ${ }^{\dagger}$ \\ Theo Siegrist, ${ }^{\dagger}$ Christian Kloc, ${ }^{\dagger}$ Zhenan Bao ${ }^{*}$,
}

\begin{abstract}
${ }^{\dagger}$ Bell Laboratories, Lucent Technologies, Murray Hill, New Jersey 07974, ${ }^{\dagger}$ Department of Materials Chemistry, Lund University, Sweden and ${ }^{\S}$ Department of Chemical Engineering, Stanford University, Stanford, California 94305
\end{abstract}

E-mail; zbao@chemeng.stanford.edu

\section{Experimental}

All chemicals were purchased from Aldrich Chemical Co. and used without further purification. The reaction apparatus was flame-dried and the reactions were carried out under a nitrogen atmosphere. ${ }^{1} \mathrm{H}$ NMR spectra were recorded on a Bruker $360 \mathrm{MHz}$ spectrometer and chemical shifts were measured relative to trimethylsilane in $\mathrm{CDCl}_{3}$ as solvent unless otherwise noted. Cyclic voltammetry (CV) was performed with a Princeton Applied Research Potentiostat model 263A interfaced to a PC computer. The solvent was dry $\mathrm{CH}_{3} \mathrm{CN}$, and the supporting electrolyte was tetrabutylammonium hexafluorophosphate $\left(\mathrm{Bu}_{4} \mathrm{NPF}_{6}\right)(0.10 \mathrm{M})$. A Pt wire was used as a counter electrode and $\mathrm{Ag} / \mathrm{AgNO}$ $(0.10 \mathrm{M})$ as a reference electrode. The reported potentials are vs the ferrocene/ferrocenium $\left(\mathrm{Fc} / \mathrm{Fc}^{+}, 4.8\right.$ V) couple, obtained by adding a crystal of ferrocene to the solution. Element analysis was performed by Roberson Microlit Laboratories, Madison, NJ. Melting points were uncorrected. Flash column chromatography was carried out using silica gel (Aldrich, 200-400 mesh). Single crystals of tetracene derivatives were grown either from solution or vapor phase.

5-Chloroteracene Anhydrous copper chloride $\left(\mathrm{CuCl}_{2}, 1.77 \mathrm{~g}, 13.2 \mathrm{mmol}\right)$ and tetracene $(3 \mathrm{~g}, 13.2$ $\mathrm{mmol})$ were placed in a flask containing chlorobenzene $(100 \mathrm{~mL})$ under $\mathrm{N}_{2}$. The mixture was stirred at $100{ }^{\circ} \mathrm{C}$ for $3 \mathrm{~h}$. After toluene $(50 \mathrm{~mL})$ was added, the resulting mixture was filtered to remove unreacted copper chloride and the solvents were removed by rotary evaporation. The solid residue was chromatographed with $\mathrm{CH}_{2} \mathrm{Cl}_{2}$ :hexane (v/v: $\left.1: 10\right)$ and subsequently recrystallized from $\mathrm{CHCl}_{3}:$ methanol (v/v: $3: 1)$ to afford platelet-shaped orange crystals $(1.20 \mathrm{~g}, 34.8 \%) . \mathrm{mp} 182{ }^{\circ} \mathrm{C} .{ }^{1} \mathrm{H}$ NMR $\delta 7.43(\mathrm{~m}, 3 \mathrm{H})$, $7.52(\mathrm{t}, 1 \mathrm{H}), 7.98(\mathrm{t}, 2 \mathrm{H}), 8.07(\mathrm{~d}, 1 \mathrm{H}), 8.47(\mathrm{~d}, 1 \mathrm{H}), 8.62(\mathrm{~s}, 1 \mathrm{H}), 8.65(\mathrm{~s}, 1 \mathrm{H}), 9.12(\mathrm{~s}, 1 \mathrm{H})$. Elemental anal. Found: C, 82.16; H, 4.21; Cl, 13.38. Calcd for $\mathrm{C}_{18} \mathrm{H}_{11} \mathrm{Cl}$ : C, 82.29; H, 4.22; Cl, 13.49 .

5-Bromotetracene In absence of light, a solution of NBS $(0.81 \mathrm{~g}, 4.38 \mathrm{mmol})$ in DMF $(25 \mathrm{~mL})$ was added dropwise to a solution of tetracene $(1 \mathrm{~g}, 4.38 \mathrm{mmol})$ in DMF $(200 \mathrm{~mL})$ at $80{ }^{\circ} \mathrm{C}$ and the reaction mixture was stirred at $100{ }^{\circ} \mathrm{C}$ for $6 \mathrm{~h}$. The mixture was poured into ice water $(500 \mathrm{~mL})$ and filtered to obtain the solids. Column chromatography with $\mathrm{CH}_{2} \mathrm{Cl}_{2}$ :hexane (v/v: 1:10) and subsequent recrystallization from toluene afforded platelet-shaped orange crystals $(0.70 \mathrm{~g}, 52.0 \%) . \mathrm{mp} 157{ }^{\circ} \mathrm{C} .{ }^{1} \mathrm{H}$ NMR $\delta 7.44(\mathrm{~m}, 3 \mathrm{H}), 7.52(\mathrm{t}, 1 \mathrm{H}), 7.98(\mathrm{t}, 2 \mathrm{H}), 8.08(\mathrm{~d}, 1 \mathrm{H}), 8.48(\mathrm{~d}, 1 \mathrm{H}), 8.66(\mathrm{~s}, 1 \mathrm{H}), 8.67(\mathrm{~s}, 1 \mathrm{H})$, 9.15 (s, 1H). Elemental anal. Found: C, 70.58; H, 3.40; Br, 26.33. Calcd for $\mathrm{C}_{18} \mathrm{H}_{11} \mathrm{Br}$ : C, 70.38; H, $3.61 ; \mathrm{Br}, 26.01$. 


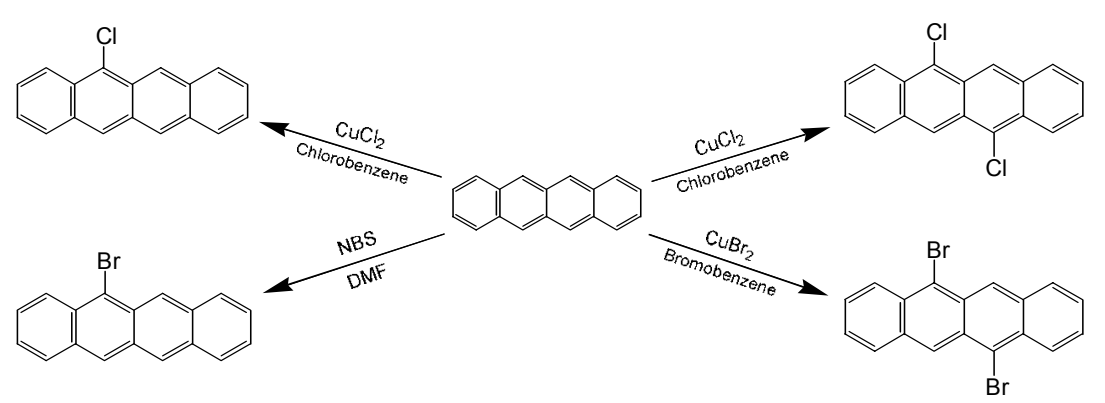

Scheme. Reactions for the synthesis of tetracene derivatives.

5,11-Dichlorotetracene This compound was synthesized with anhydrous copper chloride $\left(\mathrm{CuCl}_{2}\right)$, tetracene and chlorobenzene according to literature procedures. ${ }^{1,2} \mathrm{mp} 225{ }^{\circ} \mathrm{C} .{ }^{1} \mathrm{H}$ NMR $\delta 7.47(\mathrm{t}, 2 \mathrm{H})$, $7.56(\mathrm{t}, 2 \mathrm{H}), 8.07(\mathrm{~d}, 2 \mathrm{H}), 8.46(\mathrm{~d}, 2 \mathrm{H}), 9.12(\mathrm{~s}, 2 \mathrm{H})$. Elemental anal. Found: C, 73.01; H, 3.32; Cl, 23.48. Calcd for $\mathrm{C}_{18} \mathrm{H}_{10} \mathrm{Cl}_{2}$ : C, 72.75; H, 3.39; Cl, 23.86 .

5,11-Dibromotetracene This compound was prepared with anhydrous copper bromide $\left(\mathrm{CuBr}_{2}\right)$, tetracene and bromobenzene according to literature procedures. ${ }^{1,3}{ }^{1} \mathrm{H}$ NMR $\delta 7.48(\mathrm{t}, 2 \mathrm{H}), 7.56(\mathrm{t}, 2 \mathrm{H})$, $8.07(\mathrm{~d}, 2 \mathrm{H}), 8.47(\mathrm{~d}, 2 \mathrm{H}), 9.23(\mathrm{~s}, 2 \mathrm{H})$. Elemental anal. Found: C, 55.83; H, 2.36; Br, 41.04. Calcd for $\mathrm{C}_{18} \mathrm{H}_{11} \mathrm{Br}_{2}$ : C, 56.00; H, 2.61; $\mathrm{Br}, 41.39$.

\section{Single Crystal X-ray Data Collection}

The data collections were carried out using MoKa radiation on a Sapphire-2 CCD detector (Xcalibur 2 diffractometer, Oxford Diffraction) at room temperature. No absorption correction was applied. The integration was carried out in the CrysAlis software (Oxford Diffraction). The structure was solved using SIR-92 ${ }^{4}$ and the refinements were carried out in CRYSTALS. ${ }^{5}$ Due to the low scattering power of the crystals, the number of significant reflections available for the refinement is low. Anisotropic displacement parameters were anyway chosen for all non hydrogen atoms due to the high anisotropy. The positions of the hydrogen atoms were calculated and their parameters were constrained or fixed during the refinement.

5-Chlorotetracene (CCDC 245498) $P 2_{1} / c, a=10.7752(15) \AA, b=14.371(2) \AA, c=8.067(12) \AA, \beta=$ $93.868(11)^{\circ}$. Reflections (total/unique with $\mathrm{I}>2 \sigma$ ): $26776 / 1092$. Refinement by full least square on $\mathrm{F}$ using 172 parameters and 1092 unique reflections with final indices for reflections with $I>2 \sigma: R=3.90$, $\mathrm{w} R=4.18, \mathrm{GOF}=1.1525$, and for all reflections: $\mathrm{R}=21.12, \mathrm{wR}=14.40$. Hydrogen atoms: riding model.

5-Bromotetracene (CCDC 245497) $P 2_{1} / c, a=10.8526$ (15) $\AA, b=14.396(2) \AA, c=8.1770(12) \AA, \beta$ $=93.091(11)^{\circ}$. Reflections (total/unique with $\mathrm{I}>2 \sigma$ ): $27422 / 1575$. Refinement by full least square on $\mathrm{F}$ using 172 parameters and 1575 unique reflections with final indices for reflections with $I>2 \sigma: R=4.37$, $\mathrm{wR}=4.90, \mathrm{GOF}=1.0519$, and for all reflections: $\mathrm{R}=10.09, \mathrm{wR}=9.92$. Hydrogen atoms: riding model.

5,11-Dichlorotetracene (CCDC 245496) $P-1, a=3.8607(12) \AA, b=8.664(3) \AA, c=19.686(5) \AA, \alpha=$ $93.37(3)^{\circ}, \beta=92.02(2)^{\circ}, \gamma=99.47(3)^{\circ}$. Reflections (total/unique with $\mathrm{I}>2 \sigma$ ): $5777 / 492$. Refinement by full least square on $\mathrm{F}$ using 181 parameters and 939 unique reflections with final indices for all reflections $\mathrm{R}=9.05, \mathrm{wR}=9.85, \mathrm{GOF}=0.9959$, and for reflections with $\mathrm{I}>2 \sigma: \mathrm{R}=3.90, \mathrm{R}=4.03$, wR $=$ 7.15. Hydrogen atoms: fixed parameters.

\section{References:}

1. Tanimoto, I.; Kushioka, K.; Kitagawa, T.; Maruyama, K. Bull. Chem. Soc. Japan 1979, 52, 3586.

2. Ware, J. C.; Borchert, E. E. J. Org. Chem. 1961, 26, 2267. 
3. Mitchell, R. H.; Lai, Y.-H.; Williams, R. V. J. Org. Chem. 1979, 44, 4733.

4. Altomare, A.; Burla, M. C.; Camalli, G.; Cascarano, G.; Giacovazzo, G.; Guagliardi, A.; Polidori, G. J. Appl. Cryst. 1994, 27, 435.

5. Betteridge, P. W.; Carruthers, J. R.; Cooper, R. I.; Prout, K.; Watkins, D. J. J. Appl. Cryst. 2003, $36,1487$.

Table S1. Semi-empirical calculations with a PM3 hamiltonian in Spartan '04

\begin{tabular}{cll}
\hline & HOMO & LUMO \\
\hline Tetracene & $-7.75 \mathrm{eV}$ & $-1.24 \mathrm{eV}$ \\
5-Chlorotetracene & $-7.82 \mathrm{eV}$ & $-1.41 \mathrm{eV}$ \\
5,11-Dichlorotetracene & $-7.89 \mathrm{eV}$ & $-1.56 \mathrm{eV}$ \\
5-Bromotetracene & $-7.88 \mathrm{eV}$ & $-1.45 \mathrm{eV}$ \\
5,11-Dibromotetracene & $-8.01 \mathrm{eV}$ & $-1.65 \mathrm{eV}$ \\
\hline
\end{tabular}

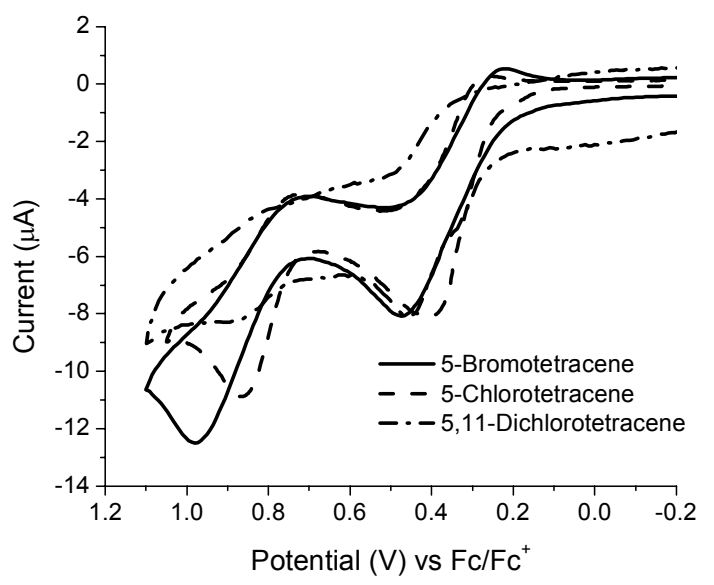

Figure S1. Cyclic voltammogram of tetracene derivatives.

Table S2. Thin film transistor performance

\begin{tabular}{|c|c|c|c|c|c|c|c|}
\hline & \multirow{2}{*}{$\begin{array}{l}\text { Substrate } \\
\text { Temp }\end{array}$} & \multicolumn{2}{|c|}{$\mathrm{SiO}_{2}$} & \multicolumn{2}{|c|}{ OTSa) treated $\mathrm{SiO}_{2}$} & \multicolumn{2}{|c|}{$\mathrm{PTS}^{\mathrm{b})}$ treated $\mathrm{SiO}_{2}$} \\
\hline & & $\begin{array}{l}\text { Mobilityc) } \\
\text { (cm²/Ns) }\end{array}$ & On/off & $\begin{array}{l}\text { Mobility } \\
\left(\mathrm{cm}^{2} / \mathrm{Ns}\right)\end{array}$ & On/off & $\begin{array}{l}\text { Mobility } \\
\left(\mathrm{cm}^{2} / \mathrm{Vs}\right)\end{array}$ & On/off \\
\hline \multirow{2}{*}{ 5-Chlorotetracene } & 0 & \multirow{2}{*}{\multicolumn{6}{|c|}{$\begin{array}{l}\text { No gate effect } \\
\text { Cloudy film }\end{array}$}} \\
\hline & 25 & & & & & & \\
\hline \multirow{2}{*}{ 5,11-Dichlorotetracene } & 0 & $3 \times 10^{-5}$ & 40 & $1 \times 10^{-3}$ & 500 & $1 \times 10^{-4}$ & 30 \\
\hline & 50 & \multicolumn{2}{|c|}{ No gate effect } & $6 \times 10^{-5}$ & $\sim 10$ & \multicolumn{2}{|c|}{ No gate effect } \\
\hline
\end{tabular}

\footnotetext{
a) Octadecyltrimethoxysilane.

b) Phenyltrimethoxysilane.

c) Calculated in saturation regions.

d) The crystallinity of all films was investigated with X-ray diffraction. Only dichlorotetracene on OTS treated $\mathrm{SiO}_{2}$ showed preferred orientation peaks at $9.6 \AA$ and $4.8 \AA$
} 